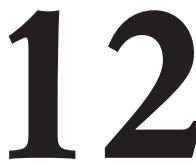

\title{
PROGRAMA PARA EL DESARROLLO DE ACTITUDES DE IGUALDAD DE GÉNERO EN CLASES DE EDUCACIÓN FÍSICA EN ESCOLARES
}

\author{
(PROGRAM FOR THE DEVELOPMENT OF ATTITUDES OF EQUALITY \\ OF GENDER IN CLASSES OF PHYSICAL EDUCATION IN SCHOOLS)
}

Antonia Pelegrín Muñoz

Universitad Miguel Hernández de Elche

Josefa María León Campos

Consejería de Educación, Formación y Empleo

Enrique Ortega Toro y Enrique Javier Garcés de Los Fayos Ruiz

Universidad de Murcia.

\section{RESUMEN}

El objetivo del estudio es intervenir en clases de educación física para el desarrollo de actitudes de igualdad en una muestra de escolares. Se administró, antes y después de la intervención, el cuestionario de actitudes sexistas en educación primaria, elaborado a partir de la Escala de Detección de Sexismo en Adolescentes (Recio, Cuadrado y Ramos, 2007). Se aplicó a 155 alumnos de entre 7 y 12 años de edad $(9,3 \pm 1,24)$, de un centro público del municipio de la Región de Murcia (España), en los meses de septiembre de 2008 y junio de 2009. La duración de la intervención se llevó a cabo en ocho sesiones que fueron distribuidas en un mes. Los resultados ponen de manifiesto, por un lado, que en los niños se detectaron más actitudes sexistas con respecto a las niñas, antes y después de la intervención. Por el otro, la efectividad de la intervención para el desarrollo de actitudes de igualdad, influyó de la misma manera en ambos géneros y edades, al disminuir dichas conductas a lo largo de un curso escolar. La actividad física y deportiva será una herramienta que permita a los profesionales trabajar la igualdad, la convivencia y valores democráticos en el contexto educativo.

\section{ABSTRACT}

The aim of this study is the analysis of the implementation of a program in physical education classes for the improvement of sexism in school. The instrument was administered before and after the implementation of the pro- 
gram, sexist attitudes questionnaire in primary education, drawn from the Sexism Scale Detection in Adolescents (Recio, Cuadrado, and Ramos, 2007). It was applied to 155 students between 7 and 12 years of age $(9,3+1,24)$, in a public institution of the municipality of Murcia (Spain), in the months of September 2008 and June 2009. The duration of the intervention was conducted in eight sessions that were distributed in a month. The results show, first, that children revealed more sexist attitudes about girls before and after the intervention. On the other hand, the effectiveness of intervention for the development of attitudes of equality, influenced in the same way in both genders and ages, and it did reduce these behaviors throughout the school year. Physical activity and sport is a tool that allows professionals to work on aspects related to equality, coexistence and democratic values in the educational context.

\section{INTRODUCCIÓN}

En la actualidad, siguen existiendo barreras que dificultan una participación físico-deportiva en la misma proporción entre niñas y niños, mujeres y hombres. La participación de las mujeres en la actividad física y en el deporte ha sido, y todavía es en la actualidad, menor que la de los hombres (Alfaro, 2004; Pelegrín, 2005). Sin embargo, existen trabajos que destacan indicadores positivos en cuanto a un aumento de la participación femenina (Alvariñas, Fernández y López, 2009; Sohaila y Dunbar, 2002; Vázquez-Gómez, 2002). Esto no es un hecho casual ni aislado, ya que la participación de la mujer es menor en los ámbitos que tradicionalmente se han considerado públicos, como el mundo laboral, deportivo, político, educativo y cultural (Hovden, 1999; Paz et al., 2011). La Escuela como una institución no es lo suficientemente potente como para luchar contra los mecanismos externos que conducen a las desigualdades de género (García del Dujo y Mínguez, 2011; Kutner, 1975). Como Vázquez-Verdera $(2010,8)$ señalaba que «La escuela no es ajena a la reproducción de los roles de género, sino que transmite lo que Madeleine Arnot llamó «códigos de género» para referirse a la organización social de la vida familiar y escolar en las que se intentan mantener en las nuevas generaciones ciertas percepciones sobre la masculinidad y la feminidad, al tiempo que se acepta como natural la jerarquía de lo masculino sobre lo femenino».

Por otro lado, la actividad física y el deporte cobran cada día mayor importancia en la vida de mujeres y hombres, al estar relacionada con aspectos fundamentales de la vida cotidiana y el bienestar, como la mejora de la salud, la relación entre las personas, el ocio y ocupación del tiempo libre, o la profesionalización y la participación en la alta competición. Sin embargo, esta evidencia no ha sido tratada con igualdad de género a lo largo de la historia del deporte (Fields, 2001; Hums y Sutton, 1999). El contexto deportivo continúa siendo un ámbito que muestra un mayor reconocimiento y 
apoyo al género masculino (Blackwood, 2010; Crawley, 1998; Lentillon, Cogerino y Kaestner, 2006; Rodríguez, Martínez y Mateos, 2004). Como MartínHorcajo $(2006,116)$ señalaba que «la dificultad mayor que sufren las mujeres que se quieren dedicar a deportes que no conforman el estereotipo femenino no es el acceso igualitario a recursos humanos, económicos y de organización, sino la falta de reconocimiento por parte de la sociedad de que las mujeres que dedican largas horas y esfuerzos a estos deportes son serias, sistemáticas, comprometidas y capaces de sacrificar, en muchas ocasiones, todo lo que haga falta para llegar al máximo de su rendimiento». Diversas investigaciones (Scraton, Pfister, Fasting y Buñuel, 1999; Menesson y Climent, 2003), revelan que la diferencia en el fútbol del nivel de juego entre hombres y mujeres, es debida principalmente a la desigualdad tan importante de recursos económicos invertidos en ambos.

Por otro lado, hay trabajos que analizan las percepciones que profesores y alumnos tienen sobre qué tipos de deportes son más adecuados según el género (Laker, Laker y Lea, 2003). Siguiendo estas creencias sociales, en los primeros Juegos Olímpicos celebrados en Atenas en 1896, las mujeres estuvieron totalmente ausentes. Sin embargo, unos años más tarde, en el 1900, participarían en golf y tenis, por considerarse deportes acordes con la naturaleza femenina (Rodríguez et al., 2004). Por tanto, la discriminación de la mujer en el deporte a lo largo de la evolución histórica, se ha centrado en una visión biologista, que ha estado fuertemente condicionada por su sexo genético para procrear y estar al cuidado de los hijos (Birke y Vines, 1987). Por todo ello, el acceso de las mujeres al ámbito deportivo ha sido tardío y ha estado lleno de dificultades. Ellas han tenido que ir superando barreras creadas por estereotipos sociales y culturales (Berg y Lahelma, 2010; LeónCampos y Pelegrín, 2008; Speer, 2002; Yu, 2009). Por ello, diversas investigaciones recogen que, las mujeres, practican menos tipos de deportes (Ezzell, 2009; McGinnis, Gentry y McQuillan, 2009), con menor frecuencia que los hombres (Alfaro, 2004; Alvariñas et al., 2009; Carlin, Salguero, Márquez y Garcés-de-Los-Fayos, 2009; Pelegrín y Garcés-de-Los-Fayos, 2007), e inclinándose por la natación, el tenis y la gimnasia, deportes que encajan en el modelo femenino tradicional.

También debemos destacar que el machismo en el deporte concierne tanto a las mujeres como a los hombres, porque cuando los hombres practican deportes como la gimnasia, son vistos como afeminados y poco viriles y son socialmente penalizados (King, 2009). Por lo tanto, el machismo tradicional empobrece la práctica deportiva, impidiendo y limitando el desarrollo de las personas (Satina, Solmon, Cothran, Loftus y Stockin-Davidson, 1998). Este aspecto crea la necesidad de desarrollar programas a edades tempranas, que incentiven la participación y elección de actividades físicodeportivas a niños y niñas por igual, para que su representación sea más 
igualitaria en el deporte de competición (Leo, García, Parejo, Sánchez y García-Mas, 2009).

Por todo ello, es fundamental trabajar una educación desde los niveles más inferiores del ámbito educativo, para fomentar los valores de igualdad y respeto ante las posibilidades y libertades de los niños y niñas en practicar el deporte que deseen, sin estereotipos sexistas que "castren» sus anhelos y sueños, solo por su género. Así, se presenta un trabajo que se centra en los siguientes objetivos: 1) Detectar actitudes sexistas en una muestra de niños y niñas de $2^{\circ}$ y $3^{\circ}$ Ciclo de Primaria a partir del género y la edad; 2) Analizar la intervención en clases de educación física para fomentar actitudes de igualdad en escolares; 3) Valorar si la intervención influye de la misma manera tanto en niños como en niñas; y 4) Evaluar la influencia de la edad en la actitud sexista de los escolares tras la intervención. Por otro lado, plantea la siguiente hipótesis: La intervención para desarrollar actitudes de igualdad en clases de educación física, mejora la variable sexismo, de manera que la mejora de la variable sexismo no depende ni del género ni de la edad del escolar.

\section{MÉTODO}

\subsection{Instrumento y procedimiento}

Se aplicó un diseño pre-experimental, con pre prueba y post prueba (Montero y León, 2007). Se administró, antes y después de la intervención, el cuestionario de actitudes sexistas en educación primaria elaborado a partir de de Detección de Sexismo en Adolescentes (Recio, Cuadrado y Ramos, 2007). Dicha escala se adaptó a un léxico que fuera comprensible y adecuado para niños y niñas de entre 7 y 12 años (véase anexos I y II).

Por otro lado, se solicitaron los permisos para la administración de los cuestionarios y fueron concedidos por la Consejería de Educación y Universidad de la Comunidad Autónoma de la Región de Murcia y el consejo escolar del centro participante del municipio de Murcia. La aplicación del cuestionario se realizó por miembros del equipo investigador en el aula del grupo, y tuvo lugar antes y después de la intervención en los meses de septiembre de 2008 y junio de 2009.

La intervención para desarrollar actitudes de igualdad forma parte de un programa más amplio, sobre el desarrollo de valores prosociales y prevención de conductas agresivas para la muestra objeto de estudio. La intervención se integró dentro de la programación didáctica de la asignatura de Educación Fí- 
sica, adaptándose a las características del centro y del alumnado. La duración de la intervención para fomentar actitudes de igualdad se llevó a cabo en ocho sesiones que fueron distribuidas en un mes. La igualdad de género en el deporte se trabajó a través de la recopilación de biografías de deportistas femeninas y masculinos de poco apoyo social con sus éxitos deportivos. El objetivo principal de la actividad fue sensibilizar y poner en conocimiento a los alumnos, la realidad de estos deportistas y los obstáculos sociales e ideológicos que se pueden encontrar en su camino profesional. Se les entregó a los alumnos una ficha con un listado de ejemplos de deportistas femeninos y masculinos para facilitar su búsqueda. Se formaron parejas de alumnos de forma arbitraria (un niño y una niña, preferentemente) y comenzaron la búsqueda por internet o por la biblioteca del centro sobre un deportista que seleccionaron previamente. Los profesores de educación física organizaron y supervisaron la actividad para que los grupos fueran heterogéneos y eligieran a un deportista diferente. Una vez que terminaron el trabajo de búsqueda e información, los alumnos realizaron unos murales donde se recogían las biografías de su deportista y lo expusieron en las paredes de los pasillos del colegio, donde fueron visitados y leídos por todos los alumnos del centro.

La codificación y análisis de los datos se hizo con el programa estadístico SPSS 17.0. Se han realizado análisis descriptivos, reflejándose las medias y las desviaciones típicas. Para conocer los efectos del programa se utilizó la prueba T para muestras apareadas, así como un análisis multivariante con medidas repetidas, en el último factor, para analizar la influencia de las variables género y ciclo en la evolución del sexismo. En todos los casos se utilizó un nivel de significación de p<.05.

\subsection{Participantes}

La muestra quedó configurada por 155 alumnos (76 niños y 79 niñas), de entre 7 y 12 años $(9,3 \pm 1,24)$. Se realizó un muestreo no probabilístico intencional, en un centro público de educación primaria del municipio de la Región de Murcia (España). La muestra ha sido seleccionada a partir de la accesibilidad y las garantías para la recogida de la información. El 46,9\% son niños y el 48,8\% niñas. A los efectos de la variable «edad», se constituyeron dos grupos: Grupo-1: 7,0-9,0 años (52,3\%) y Grupo-2: 10-12 años $(47,7 \%)$.

\section{RESULTADOS}

En la tabla 1 se aprecian las medias y desviaciones típicas de la variable sexismo de los datos obtenidos en la medición inicial (pre-test) y en la medición final (pos-test), de los participantes objeto de estudio. 
En primer lugar cabe que los niños presentaron valores más altos de sexismo que las niñas $\left(\mathrm{t}_{91}=3.763, \mathrm{p}<.001\right)$ en la medida inicial. Estas diferencias también se aprecian en la medida final $\left(\mathrm{t}_{91}=3.738, \mathrm{p}<.001\right)$. Por el contrario no se apreciaron diferencias entre ciclos ni en la medida inicial $\left(t_{91}=2.177, p=.079\right)$, ni en la medida final $\left(t_{91}=1.373, p=.173\right)$.

Por otro lado se aprecian disminuciones estadísticamente significativas entre la medida inicial y la medida final en el total de la muestra $\left(t_{92}=4.978\right.$, $\mathrm{p}<.001)$, en chicos $\left(\mathrm{t}_{39}=3.009, \mathrm{p}<.01\right)$, en chicas $\left(\mathrm{t}_{52}=4.031, \mathrm{p}<.001\right)$, en segundo ciclo $\left(\mathrm{t}_{44}=4.380, \mathrm{p}<.001\right)$ y en tercer ciclo $\left(\mathrm{t}_{47}=2.793, \mathrm{p}<.01\right)$. De este modo, los resultados indican que, tras la aplicación de la intervención, se observaron menos actitudes y conductas sexistas en niños y niñas de segundo y tercer ciclo de primaria.

\begin{tabular}{|c|c|c|c|c|c|c|c|c|c|c|}
\hline \multirow{4}{*}{$\begin{array}{l}\text { Pre- } \\
\text { test } \\
\text { Post- } \\
\text { test }\end{array}$} & \multicolumn{2}{|c|}{$\begin{array}{c}\text { Muestra } \\
\text { total }(n=93)\end{array}$} & \multicolumn{2}{|c|}{ Chicos $(n=40)$} & \multicolumn{2}{|c|}{ Chicas $(n=53)$} & \multicolumn{2}{|c|}{$\begin{array}{c}\text { Segundo Ciclo } \\
(\mathrm{n}=45)\end{array}$} & \multicolumn{2}{|c|}{$\begin{array}{l}\text { Tercer Ciclo } \\
\quad(n=48)\end{array}$} \\
\hline & Media & $\begin{array}{l}\text { Desv. } \\
\text { típica }\end{array}$ & Media & $\begin{array}{c}\text { Desv. } \\
\text { típica }\end{array}$ & Media & $\begin{array}{l}\text { Desv. } \\
\text { típica }\end{array}$ & Media & $\begin{array}{c}\text { Desv. } \\
\text { típica }\end{array}$ & Media & $\begin{array}{l}\text { Desv. } \\
\text { típica }\end{array}$ \\
\hline & 15.94 & 4.26 & 17.73 & 4.67 & 14.59 & 3.38 & 16.87 & 4.35 & 15.06 & 4.02 \\
\hline & $14.19 * *$ & 4.14 & $15.93 *$ & 4.59 & $12.89 * *$ & 3.25 & $14.80 * *$ & 4.35 & $13.63 *$ & 3.91 \\
\hline
\end{tabular}

Tabla 1. Datos descriptivos de Sexismo.

Con objeto de conocer si el género del alumno influye en la mejora de la variable sexismo se aplicó un análisis de la varianza de dos factores $(2 \times 2)$, género y momento de la medición, con medidas repetidas en el último factor. Se apreció que el efecto de la interacción del factor momento de la medición $x$ el género no era significativo $\left(F 1_{91}=0.21, p=.886\right)$, por lo que puede afirmarse que el género no afecta a los cambios producidos en el sexismo; es decir, que la intervención influye positivamente independientemente del género.

Con respecto a la posible influencia del ciclo educativo al que pertenece el alumno en la mejora de la variable sexismo, se aprecia que el efecto de la interacción del factor momento de la medición x el ciclo no es significativo $\left(F 1_{, 91}=0.806, \mathrm{p}=.372\right)$, por lo que también puede afirmarse que la per- 
tenencia a un ciclo educativo o a otro no afecta a los cambios producidos en el sexismo. Por tanto, la intervención afecta de la misma manera independientemente del ciclo al que pertenece el alumno.

\section{CONCLUSIONES Y DISCUSIÓN}

Los objetivos de este trabajo han sido, por un lado, conocer las actitudes sexistas en una muestra de niños y niñas de $2^{\circ}$ y $3^{\circ}$ Ciclo de Primaria a partir del género y la edad y, por el otro, analizar la intervención en clases de educación física para fomentar actitudes de igualdad. Los resultados encontrados ponen de manifiesto que, los niños, presentaron más actitudes sexistas con respecto a las niñas, antes y después de la intervención. Estos datos indicaron creencias más sexistas en los niños, en los rasgos adjudicados tradicionalmente a lo femenino y a lo masculino como, por ejemplo, la creencia de que las niñas son más débiles y menos inteligentes. También más actitudes sexistas en las capacidades de cada género para desempeñar determinados roles y funciones como, por ejemplo, la creencia de que habría menos paro si las mujeres no trabajaran, el lugar de la mujer es el hogar para estar al cuidado de sus hijos o que el padre es el que tiene que tomar las decisiones más importantes de su familia. Y más actitudes sexistas en cuanto a percibir a las niñas como menos hábiles en los deportes. Estos resultados nos llevan a la reflexión de que, en la actualidad, continúan observándose los síntomas de una educación más sexista en los niños que en las niñas, donde los estereotipos de género siguen estando muy presentes, aspecto que también se recoge en el trabajo de Díaz-Aguado y Martín (2011). Estas conductas se podrían explicar por la presencia e influencia de modelos parentales que las transmiten de generación en generación, en el marco de una sociedad que contribuye a la formación de estos estereotipos (VázquezVerdera, 2010).

Además, estas creencias y actitudes pueden estar repercutiendo en una menor participación de la mujer en el deporte, limitando así sus posibilidades para desarrollar su habilidad física (Blackwood, 2010; Hovden, 1999; Lentillon et al., 2006), y de favorecer la categorización de deportes considerados como masculinos y femeninos. Todas estas barreras que dificultan una participación físico-deportiva igualitaria entre niñas y niños (Berg y Lahelma, 2010; León-Campos y Pelegrín, 2008; Speer, 2002; Yu, 2009), generan la necesidad de intervenir a través de programas que se incluyan en el currículum escolar de los alumnos. En este sentido, en el trabajo de Alvariñas et al., (2009), se concluyó que alumnos de Educación Secundaria pensaban que las chicas pueden realizar cualquier tipo de deporte, pero que hay deportes más apropiados para chicos que para chicas, y viceversa. También, el trabajo de Blández, Fernández y Sierra (2007) llegó a conclusiones similares, tanto 
con alumnos de Educación Primaria como de Secundaria. En nuestro trabajo, esta realidad se pudo observar al comprobar que, tras la recopilación de biografías de deportistas femeninas y masculinos de poco apoyo social con sus éxitos deportivos, continuaban apareciendo numerosas fotografías de deportistas famosos masculinos, de modalidades deportivas con un mayor número de aficionados.

Por otro lado, se ha comprobado la efectividad de la intervención para la mejora de la variable sexismo, que influyó de la misma manera en ambos géneros y ciclos, al generar cambios a lo largo de un curso escolar. De este modo, las conductas de igualdad aumentaron significativamente en opiniones relacionadas con las posibilidades de que, hombres y mujeres, puedan desempeñar funciones y roles similares en el trabajo, el deporte y el hogar. Por ello, se genera la necesidad de seguir trabajando en esta realidad social desde la infancia y la niñez (Díaz-Aguado y Martín, 2011), para fomentar la igualdad de género y ofrecer modelos alternativos, que permitan desarrollar valores de igualdad a una cultura en la que, la figura masculina, continúa estando por encima de la femenina (Blackwood, 2010; Lentillon et al., 2006). La escuela, constituye un espacio privilegiado de socialización y de formación en valores que permite, tanto a alumnos como a alumnas, trabajar las desigualdades de género, pero aún no es lo suficientemente potente como para luchar contra los factores de riesgo relacionados (García del Dujo y Mínguez, 2011; Kutner, 1975; Vázquez-Verdera, 2010).

\section{CONCLUSIONES}

Por último, a partir de los resultados encontrados en nuestro trabajo, a continuación presentamos las principales conclusiones, concretamente:

- Los niños mostraron niveles más altos de conductas sexistas antes y después de las actividades realizadas.

- Las conductas de igualdad aumentaron significativamente tras la intervención, tanto en niños como en niñas, de edades comprendidas entre los 7 y los 12 años, a la largo de un curso escolar.

- Se concluye, por tanto, que la intervención en clases de educación física para desarrollar actitudes y conductas de igualdad en escolares, ha influido muy positivamente en las opiniones sobre la igualdad de género, disminuyendo considerablemente las conductas sexistas en escolares de ambos géneros, de segundo y tercer ciclo de educación primaria. Sin embargo, a pesar de que los efectos de la intervención influyeran positivamente y por igual, tanto en los niños como en las 
niñas, aquéllos mostraron niveles más altos de conductas sexistas, antes y después de las actividades realizadas.

- Es fundamental sensibilizar a la comunidad de esta realidad que sufren las mujeres deportistas, estableciendo programas de intervención en el ámbito escolar. Para ello, será necesario promover una educación no sexista y en pro igualdad de oportunidades desde edades tempranas. Además, son importantes los factores psicológicos, educativos y socioculturales que influyen en su aprendizaje, así como el papel que juegan las mujeres deportistas como modelos. Por tanto, habrá que establecer un deporte escolar de igualdad, que dé respuesta a todos los niños independientemente de su género.

- Niños y adolescentes encontrarán en el deporte, no sólo un medio que les permita adquirir y potenciar las habilidades físicas y técnicas, sino también un contexto enriquecedor de experiencias positivas.

- Consideramos que se ha de hacer hincapié en la necesidad de trabajar la base emocional y social de los niños y niñas, permitiendo que adquieran habilidades para una mejor convivencia con los demás, así como para potenciar el desarrollo de la igualdad.

En cuanto a las limitaciones del trabajo, hay que matizar que los resultados no han sido más favorables, probablemente debido a que hubieran sido necesarias más sesiones que incluyeran actividades relacionadas con la igualdad. Otro de los aspectos que hay que tener en cuenta en futuras investigaciones, es la importancia de la implicación de los docentes, así como su estilo pedagógico en el desarrollo de las clases, puesto que este aspecto puede influir positivamente o no en los cambios deseados de los alumnos y alumnas, al considerarse los profesores como uno de los agentes socializadores más significativos (González y Campos, 2010; Romance, Weiss y Bockowen, 1986). También destacar que, una condición ligada a unos resultados favorables, es la formación previa de los docentes implicados en la intervención, ya que ésta va a fomentar su implicación y clarificar nuestros objetivos para hacerlos también suyos. Por último, los resultados obtenidos no han podido ser contrastados con otros estudios similares realizados en población española. Por ello, las líneas de futuros trabajos podrían dirigirse a llevar a cabo la intervención en otros centros escolares de educación primaria, tanto de la misma comunidad autónoma como de otras, para comprobar si los resultados son generalizables a otros alumnos y a otras características de centro. 


\section{NOTAS}

Esta investigación ha sido financiada por la Universidad de Murcia y la Consejería de Educación, Formación y Empleo de la Comunidad Autónoma de la Región de Murcia. Nuestro agradecimiento al centro educativo participante. 


\section{REFERENCIAS BIBLIOGRÁFICAS}

Alfaro, E. (2004). El talento psicomotor y las mujeres en el deporte de alta competición. Revista de Educación, 335, 127-151.

Alvariñas, M.; Fernández, M. A. y López, C. (2009). Actividad física y percepciones sobre deporte y género. Revista de Investigación en Educación, 6, 113-122.

Berg, P. y Lahelma, E. (2010). Gendering processes in the field of physical education. Gender and Education, 22 (1), 31-46.

Birke, L. I. A. y Vines, G. (1987). A Sporting Chance: The Anatomy of Destiny? Women's Studies International Forum, 10 (4), 337-347.

Blackwood, T. (2010). Playing baseball/playing 'house': The reproduction and naturalization of 'separate spheres' in Japanese high school baseball. Sport, Education and Society, 15 (1), 83-101.

Blández, J.; Fernández, E. y Sierra, M, A. (2007). Estereotipos de género, actividad física y escuela: La perspectiva del alumnado. Profesorado. Revista de currículum y formación del profesorado [en línea], 11, 2. Disponible en: http://www.ugr.es/local/recfpro/rev112A RT5.pdf [consulta 2010, 4 de abril].

Carlin, M.; Salguero, A.; Márquez, S. y Garcés-de-Los-Fayos, E. J. (2009). Análisis de los motivos de retirada de la práctica deportiva y su relación con la orientación motivacional en deportistas universitarios. Cuadernos de Psicología del Deporte, 9 (1), 85-99.

Crawley, S. L. (1998). Gender, Class and the Construction of Masculinity in Professional Sailing: A Case Study of the America3 Women's Team. Internation- al Review for the Sociology of Sport, 33 (1), 33-42.

Díaz-Aguado, M. J. y Martín, G. (2011). Convivencia y aprendizaje escolar en la adolescencia desde una perspectiva de género. Psicothema, 23 (2), 252-259.

Ezzell, M. B. (2009). «Barbie Dolls» on the Pitch: Identity Work, Defensive Othering, and Inequality in Women's Rugby. Social Problems, 56 (1), 111-131.

Fields, S. K. (2001). Cultural Identity, Law, and Baseball. Culture, Sport, Society, 4 (2), 23-42.

García del Dujo, A. y Mínguez, R. (2011). Los límites de la educación en valores cívicos: Cuestiones y propuestas pedagógicas. Educación XX1, 14 (2), 263284.

González, M. D. y Campos, A. (2010). La intervención didáctica del docente del deporte escolar, según su formación inicial. Revista de Psicodidáctica, 15 (1), 101-120.

Hovden, J. (1999). When Women Become "Sand in the Machinery» Gender, Interaction and Power in DecisionMaking Processes in Sports Organizations. Sosiologisk tidsskrift, 7 (3), 209-233.

Hums, M. A. y Sutton, W. A. (1999). Women Working in the Management of Professional Baseball: Getting to First Base? Journal of Career Development, 26 (2), 147-158.

King, S. (2009). Virtually : Mark Bingham, the War on Terror, and the Sexual Politics of Sport. Journal of Sport and Social Issues, 33 (1), 5-24.

Kutner, J. R. (1975). Sex Discrimination in Athletics. Villanova Law Review, 21 (5), 876-903. 
Laker, A.; Laker, J. C. y Lea, S. (2003). School Experience and the Issue of Gender. Sport, Education and Society, 8 (1), 73-89.

Lentillon, V.; Cogerino, G. y Kaestner, M. (2006). Injustice in physical education: gender and the perception of deprivation in grades and teacher support. Social Psychology of Education, 9 (3), 321-339.

Leo, F. M.; García, T.; Parejo, I.; Sánchez, P. A. y García-Mas, A. (2009). Aplicación de un programa de intervención para la mejora de la cohesión y la eficacia en jugadores de baloncesto. Cuadernos de Psicología del Deporte, 9 (1), 73-84.

León-Campos, J. M. y Pelegrín, A. (2008). ¿Sexismo en el deporte?, en Díaz, J., Díaz, I. y Dosil, J. (eds) La psicología del Deporte en Iberoamérica. Actas del $2^{\circ}$ congreso de la Sociedad Iberoamericana de Psicología del Deporte. Torrelavega, Cantabria, España, 356357.

Martín-Horcajo, M. (2006). Contribución del feminismo de la diferencia sexual a los análisis de género en el deporte. Revista Internacional de Sociología, 44, 111-131.

McGinnis, L. P.; Gentry, J. W. y McQuillan, J. (2009). Ritual-based Behavior that Reinforces Hegemonic Masculinity in Golf: Variations in Women Golfers' Responses. Leisure Sciences, 31 (1), 19-36.

Menesson, C. y Clement, J. P. (2003). Homosociability and homosexuality: The case of soccer played by women. International Review for the Sociology of Sport, 38, 311-330.

Montero, y León, O. G. (2007). Guía para nombrar los estudios de investigación en Psicología. International Journal of
Clinical and Health Psychology, 7 (3), 847-862.

Paz, M.; Guillén-Riquelme, A.; GómezGarcía, A.; Quevedo-Blasco, R.; Sierra, J. C. y Buela-Casal, G. (2011). Análisis del rendimiento en el doctorado en función del sexo. Educación XX1, 14 (1), 17-33.

Pelegrín, A. (2005). Variables de riesgo asociadas a la salud mental del deportista profesional y de alto rendimiento, en Mora, J. A.; Chapado de la Calle, F. (eds) Visión Actual de la Psicología del Deporte. Sevilla: Wanceulen, 466-483.

Pelegrín, A. y Garcés de Los Fayos, E. J. (Eds) (2007). Agresión y violencia en el deporte. Sevilla: Wanceulen.

Recio, P.; Cuadrado, I. y Ramos, E. (2007). Propiedades psicométricas de la escala de detección de sexismo en adolescentes (DSA). Psicothema, 19 (3), 522-528.

Rodríguez, D.; Martínez, M. J. y Mateo, C. (2005). Identidad y estereotipos de la mujer en el deporte. Una aproximación a la evolución histórica. Revista de Investigación en Educación, 2, 109 - 126. Disponible en: http://webs.uvigo.es/reined/ejemplares/2/reined_02_06.pdf [consulta: 2010, 6 de abril].

Romance, T. J.; Weiss, M. R. y Bockowen, J. (1986). A program to promote moral development through elementary school physical education. Journal of Teaching Physical Education, 5, 125-136.

Satina, B.; Solmon, M. A.; Cothran, D. J.; Loftus, S. J. y Stockin-Davidson, K. (1998). Patriarchal Consciousness: Middle School Students' and Teachers' Perspectives of Motivational Practices. Sport, Education and Society, 3 (2), 181-200.

Scraton, S., Pfister, G., Fasting, K. y Buñuel, A. (1999). It`s still a man`s 
game? The experiences of top-level European women footballers. International Review for the Sociology of Sport, 34, 99-111.

Sohaila, S. y Dunbar, M. D. (2002). The Social Construction of Female and Male High School Basketball Participation: Reproducing the Gender Order through a Two-Tiered Sporting Institution. Sociological Perspectives, 45 (4), 353-378.

Speer, S. A. (2002). Sexist Talk: Gender Categories, Participants' Orientations and Irony. Journal of Sociolinguistics, 6 (3), 347-377.
Vázquez-Verdera, V. (2010). La perspectiva de la ética del cuidado: Una forma diferente de hacer educación. Educación XX1, 13 (1), 177-197.

Vázquez-Gómez, B. (2001). La cultura física y las diferencias de género en el umbral del siglo XXI, en Devís, J. (Ed) La educación física, el deporte y la salud en el siglo XXI. Alicante, España: Marfil, 213-218.

Yu, C. C. (2009). A Content Analysis of News Coverage of Asian Female Olympic Athletes. International Review for the Sociology of Sport, 44 (2), 283305. 


\section{PALABRAS CLAVE}

Discriminación sexual, educación física, escuela primaria, desigualdad social, intervención.

\section{KEY WORDS}

Sex discrimination, physical education, primary school, social inequality, intervention.

\section{PERFIL ACADÉMICO DE LOS/LAS AUTORES/AS}

Antonia Pelegrín Muñoz. Profesora Contratado Doctor del Departamento de Psicología de la Salud de la Universidad Miguel Hernández de Elche. Doctora y Licenciada en Psicología. Máster en Psicología de la Actividad Física y el Deporte. Autora de publicaciones sobre el análisis y la intervención del comportamiento agresivo en niños y adolescentes.

Josefa María León Campos. Maestra Interina de Pedagogía Terapéutica de la Comunidad Autónoma de la Región de Murcia. Licenciada en Pedagogía y Máster en Audición y Lenguaje. Doctoranda en el Departamento de Psicología de la Salud de la Universidad Miguel Hernández de Elche. Su línea de investigación se centra en la implantación de programas para el desarrollo de valores prosociales en el contexto educativo.

Enrique Ortega Toro. Profesor Titular de Universidad en el área de conocimiento Didáctica de la Expresión Corporal de la Facultad de Ciencias del Deporte de la Universidad de Murcia. Doctor y Licenciado en Educación Física. Autor de numerosos artículos, libros e investigaciones en los ámbitos de la iniciación deportiva, aspectos psicológicos y análisis de las acciones deportivas.

Enrique Javier Garcés de Los Fayos Ruiz. Profesor Titular del Departamento de Personalidad, Evaluación y Tratamientos Psicológicos de la Universidad de Murcia. Doctor y Licenciado en Psicología. Autor de artículos, libros e investigaciones en psicología del deporte. Sus líneas de investigación se centran en el burnout en deportistas.

Dirección de los autores: Departamento Psicología de la Salud.

Universidad Miguel Hernández de Elche.

Avda. de la Universidad, s/n, Edificio Altamira 03202, Elche (Alicante).

E-mail: apelegrin@umh.es 
Fecha Recepción del Artículo: 19. Noviembre. 2010

Fecha Revisión del Artículo: 11. Marzo. 2011

Fecha Aceptación del Artículo: 19. Mayo. 2011

Fecha de Revisión para publicación: 18. Noviembre. 2011 


\begin{tabular}{|c|c|c|c|c|c|c|}
\hline \multicolumn{7}{|c|}{$\begin{array}{c}\text { Anexo I } \\
\begin{array}{c}\text { DSA. Escala de Detección de Sexismo en Adolescentes* (E. Ramos, I. Cuadrado y } \\
\text { P. Recio). }\end{array} \\
\text { Marca con una cruz tu grado de Acuerdo o Desacuerdo con cada una de las si- } \\
\text { guientes frases, teniendo en cuenta la siguiente escala: } \\
\text { 1. Totalmente en desacuerdo; 2. Bastante en desacuerdo; 3. Algo en desacuerdo; } 4 . \\
\text { Algo de acuerdo; 5. Bastante de acuerdo; 6. Totalmente de acuerdo. }\end{array}$} \\
\hline & 1 & & & & & 6 \\
\hline \multicolumn{7}{|l|}{$\begin{array}{l}\text { 1. Las mujeres son, por naturaleza, } \\
\text { más pacientes y tolerantes que los } \\
\text { hombres. }\end{array}$} \\
\hline \multicolumn{7}{|l|}{$\begin{array}{l}\text { 2. El lugar más adecuado para la } \\
\text { mujer es su casa con su familia. }\end{array}$} \\
\hline \multicolumn{7}{|l|}{$\begin{array}{l}\text { 3. El afecto y el cariño son más im- } \\
\text { portantes para las mujeres que para } \\
\text { los hombres. B; rasgo }\end{array}$} \\
\hline \multicolumn{7}{|l|}{$\begin{array}{l}\text { 4. Las mujeres son más débiles que } \\
\text { los hombres en todos los aspectos. } \\
\text { Rasgo }\end{array}$} \\
\hline \multicolumn{7}{|l|}{$\begin{array}{l}\text { 5. Una medida positiva para acabar } \\
\text { con el paro sería que las mujeres se } \\
\text { quedaran en casa. }\end{array}$} \\
\hline \multicolumn{7}{|l|}{$\begin{array}{l}\text { 6. Las mujeres están mejor dotadas } \\
\text { que los hombres para complacer a } \\
\text { los demás (estar atentas a lo que } \\
\text { quieren y necesitan). B; rasgo }\end{array}$} \\
\hline \multicolumn{7}{|l|}{$\begin{array}{l}\text { 7. Es más natural que sean las hijas } \\
\text { y no los hijos las que se hagan cargo } \\
\text { de los padres ancianos. }\end{array}$} \\
\hline \multicolumn{7}{|l|}{$\begin{array}{l}\text { 8. Por su mayor sensibilidad, las } \\
\text { mujeres son más compasivas que } \\
\text { los hombres hacia su pareja. B; ras- } \\
\text { go }\end{array}$} \\
\hline $\begin{array}{l}\text { 9. Atender bien la casa es obliga- } \\
\text { ción de la mujer. }\end{array}$ & & & & & & \\
\hline
\end{tabular}




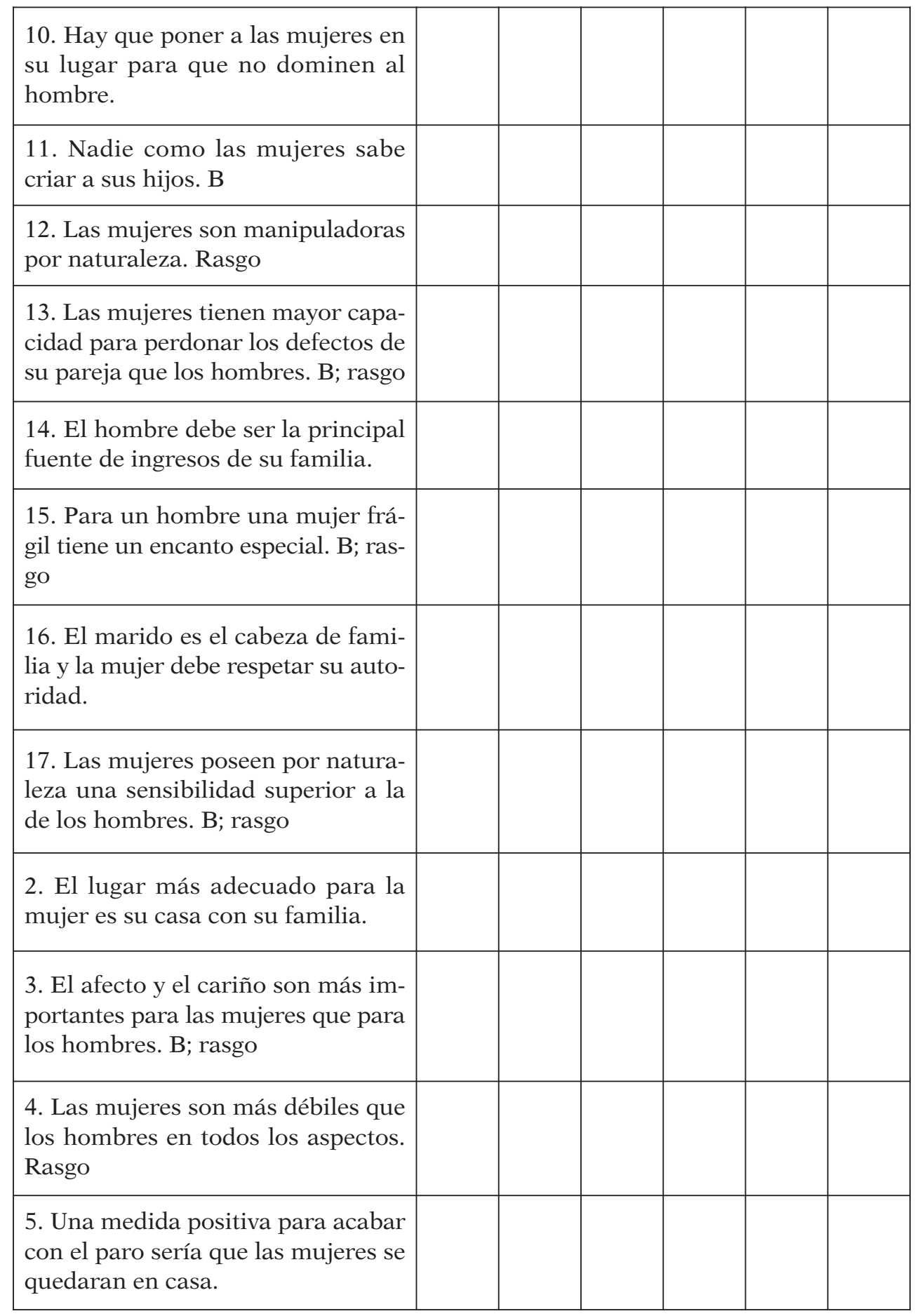




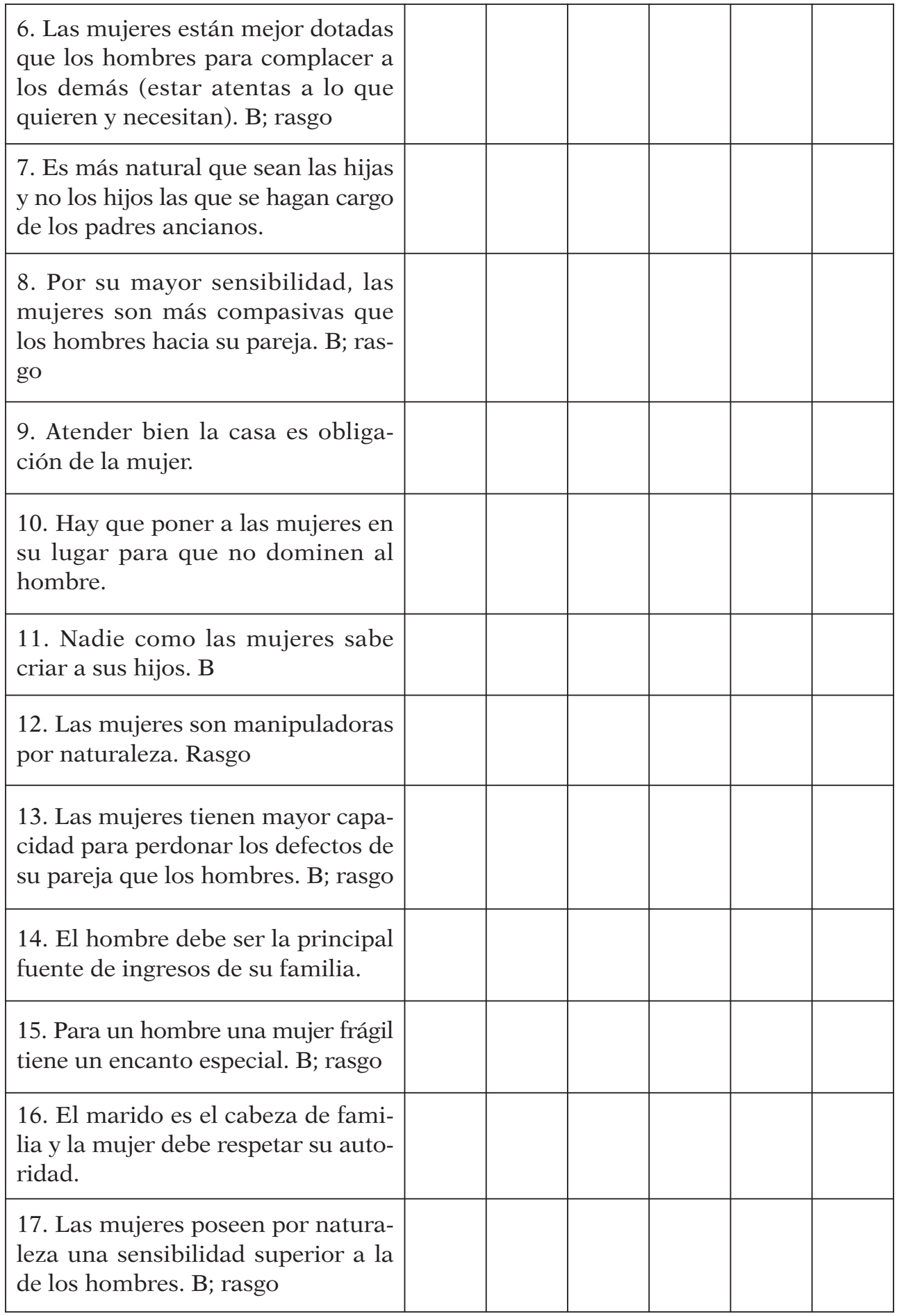




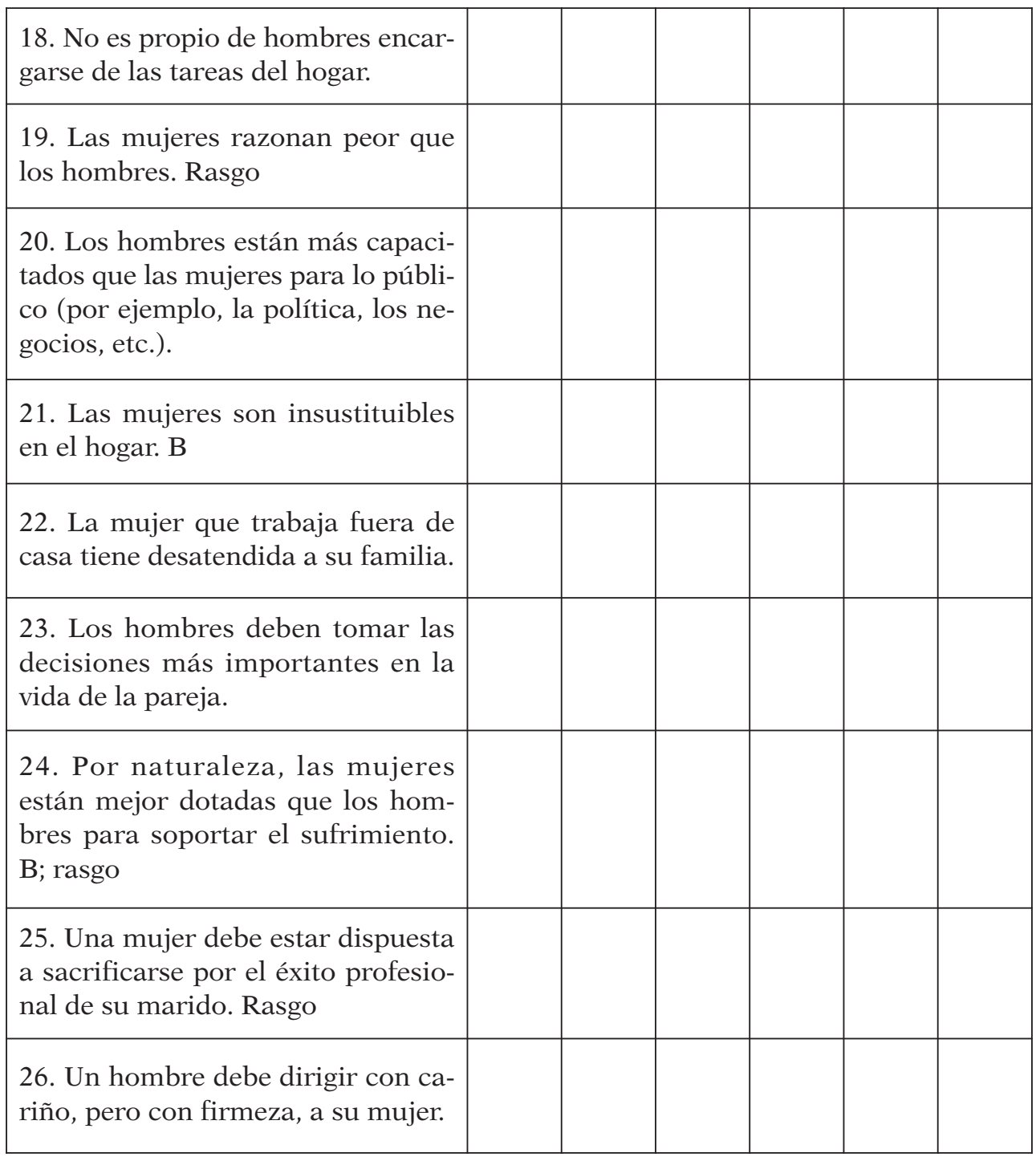

*Los ítems en los que aparece la letra B miden sexismo benévolo, el resto miden sexismo hostil. Se señalan los ítems destinados a medir creencias sexistas sobre los rasgos adjudicados tradicionalmente a lo femenino y a lo masculino. El resto miden creencias sexistas sobre la supuesta aptitud de cada sexo para desempeñar determinados roles y funciones. 


\begin{tabular}{|c|c|}
\hline $\begin{array}{l}\text { Anexo II } \\
\text { Cuestionario de Actitudes Sexistas en Educación Primaria elaborad } \\
\text { Escala de Detección de Sexismo en Adolescentes* (Recio, Cuadrado y } \\
\text { A continuación, haz un círculo en la respuesta que indica lo q }\end{array}$ & $\begin{array}{l}\text { a partir de la } \\
\text { Ramos, 2007). } \\
\text { ue haces. }\end{array}$ \\
\hline 1. Las chicas son más débiles que los chicos en general. Rasgo & Sí / No \\
\hline 2. Habría menos paro si las mujeres no trabajaran. Rol & Sí / No \\
\hline 3. El lugar de la mujer es el hogar. Rol & Sí / No \\
\hline $\begin{array}{l}\text { 4. Una chica debería poder hacer las mismas cosas que un chico } \\
\text { (p.e., jugar al fútbol, jugar al video juego, quedar con las amigas, } \\
\text { etc.). Deporte }\end{array}$ & Sí / No \\
\hline 5. Las mamás son las únicas que saben criar a los hijos. Rol & Sí / No \\
\hline 6. Las chicas perdonan más fácilmente que los chicos. Rasgo & Sí / No \\
\hline 7. Papá debe traer el dinero a su familia. Rol & Sí / No \\
\hline $\begin{array}{l}\text { 8. Papá es el jefe de la familia y la madre debe respetar lo que él } \\
\text { diga. Rol }\end{array}$ & Sí / No \\
\hline $\begin{array}{l}\text { 9. Papá realiza las tareas del hogar (p.ej.: limpiar cristales, planchar, } \\
\text { lavar,...). Rol }\end{array}$ & Sí / No \\
\hline 10. Las chicas deben ayudar a sus madres en las tareas del hogar. & Rol Sí / No \\
\hline $\begin{array}{l}\text { 11. Las mamás son las únicas que saben ocuparse de las tareas del } \\
\text { hogar. Rol }\end{array}$ & Sí / No \\
\hline 12. Las mamás que trabajan tienen desatendida a su familia. Rol & Sí / No \\
\hline 13. Papá debe tomar las decisiones más importantes de su familia. & Rol Sí / No \\
\hline 14. Las chicas aguantan mejor el dolor. Rasgo & Sí / No \\
\hline 15. Las chicas son más torpes en los deportes. Deporte & Sí / No \\
\hline 16. Los chicos son buenos en los deportes. Deporte & Sí / No \\
\hline $\begin{array}{l}\text { 17. Está bien que las mamás mantengan económicamente a sus fa- } \\
\text { milias. Rol }\end{array}$ & Sí / No \\
\hline $\begin{array}{l}\text { 18. Las chicas deberían tener las mismas oportunidades de trabajo que } \\
\text { los chicos. Rol }\end{array}$ & Sí / No \\
\hline
\end{tabular}




\begin{tabular}{|c|c|}
\hline 19. Las chicas pueden ser muy buenas jugando al fútbol. Deporte & Sí / No \\
\hline $\begin{array}{l}\text { 20. Las madres son las que deben cuidar a los hijos cuando están en- } \\
\text { fermos. Rol }\end{array}$ & Sí / No \\
\hline 21. Se necesitan más mujeres que sean jefas en los trabajos. Rol & Sí / No \\
\hline 22. Es mejor que las mamás cambien los pañales a sus hijos. Rol & Sí / No \\
\hline $\begin{array}{l}\text { 23. Si mamá trabaja las tareas de casa deberían repartirse con el pa- } \\
\text { dre. Rol }\end{array}$ & Sí / No \\
\hline 24. Los chicos pueden ser muy buenos bailarines. Deporte & Sí / No \\
\hline $\begin{array}{l}\text { 25. Los hombres están más preparados para los negocios y la polí- } \\
\text { tica que las mujeres. Rasgo }\end{array}$ & Sí / No \\
\hline 26. A las chicas les gusta mandar en los juegos. Deporte & Sí / No \\
\hline
\end{tabular}

*Los ítems que están en cursiva han sido añadidos 
